\title{
Collagenous Colitis in a Japanese Woman: Successful Treatment with Mesalazine
}

\author{
Keiko Suzuki $^{\mathrm{a}}$ Taiichi Kawabe $^{\mathrm{a}}$ Hirokazu Takahashi $^{\mathrm{a}}$ Satoshi Nakao ${ }^{\mathrm{a}}$ Akihiro Suzuki $^{\mathrm{a}}$ \\ Masahiko Inamori $^{a}$ Hiroshi lida ${ }^{a}$ Hiroki Endo $^{a}$ Tomoyuki Akiyama ${ }^{a}$ Koji Fujita $^{a}$ \\ Masato Yoneda ${ }^{a}$ Yasunobu Abe ${ }^{a}$ Noritoshi Kobayashi ${ }^{a}$ Kensuke Kubota ${ }^{a}$ \\ Hisashi Oshiro $^{b}$ Atsushi Nakajima ${ }^{a}$ \\ ${ }^{a}$ Gastroenterology Division, and ${ }^{b}$ Pathology, Yokohama City University School of Medicine, Yokohama, Japan
}

\section{Dear Sir,}

An 80-year-old Japanese woman was admitted to our hospital with a 4-month history of diarrhea and weight loss (she lost $8 \mathrm{~kg}$ during this period). Laboratory data were almost normal. Upper and lower endoscopic examination and radiological enteroclysis revealed no specific findings. Biopsies were taken from the colon and rectum. From seven samples, none of them showed cryptitis, crypt abscess, alternation of crypt or hyperplasia. Congo red staining was also negative. All specimens stained with the Masson trichrome method revealed thickening of the subepithelial collagen layer beneath the basal membrane, inflation of the lamina propria and epithelial lesions with intraepithelial lymphocytes (fig. 1). We made a diagnosis of collagenous colitis and mesalazine therapy ( $1.5 \mathrm{~g} /$ day $)$ was started. As the patient was elderly, we did not choose prednisolone treatment. The symptoms resolved 1 week after the procedure.

Microscopic colitis is a disease defined as those entities characterized by chronic watery non-bloody diarrhea, abdominal pain, normal radiological and endoscopic appearances and microscopic abnormalities in the colon [1]. Microscopic colitis

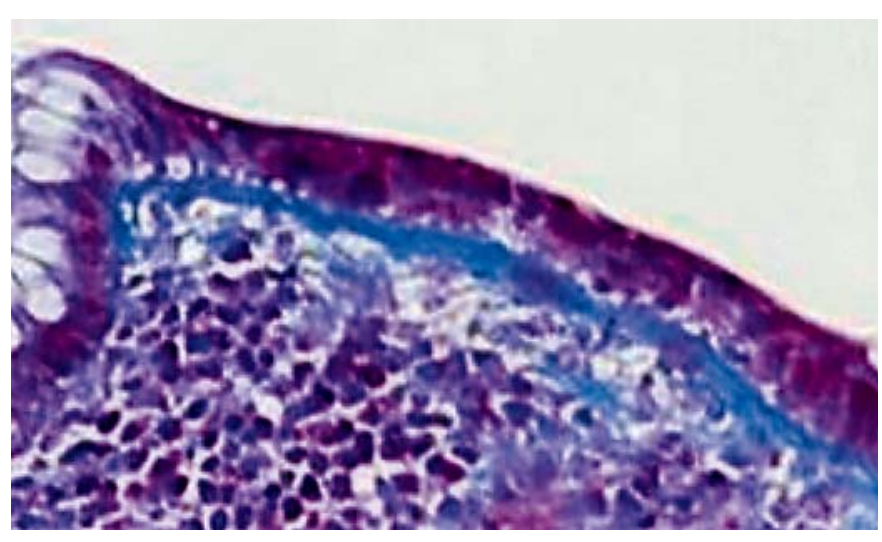

Fig. 1. Biopsy from colon showing increased subepithelial collagen layer (blue in this picture), inflation of lamina propria and epithelial lesions with intraepithelial lymphocytes.

mainly includes two diseases: collagenous colitis and lymphocytic colitis. The diagnosis relies on specific microscopic changes seen in colonic mucosal biopsies. Collagenous colitis was first described in 1976 by Lindström and the typical patient is a middle-aged or elderly woman, with the female-to-male ratio approximately $7: 1$.
The etiology is unknown [2]. Collagenous colitis differs from lymphocytic colitis by a specific histopathological feature consisting of the presence of a band of subepithelial collagen $(10 \mu \mathrm{m}$ or more, the normal basement membrane being about 3 $\mu \mathrm{m}$ thick) adjacent to the basal membrane, and inflammatory changes can be identi-

\section{KARGER}

Fax +4161306 1234 E-Mail karger@karger.ch www.karger.com
(C) 2008 S. Karger AG, Basel 0012-2823/08/0774-0155\$24.50/0

Accessible online at: www.karger.com/dig
Masahiko Inamori

Yokohama City University, School of Medicine, Gastroenterology Division

3-9 Fukuura, Kanazawa-ku, Yokohama, Kanagawa 236-0004 (Japan)

Tel. +81 457872640 , Fax +81 457843546

E-Mail inamorim@med.yokohama-cu.ac.jp 
fied in the lamina propria together with superficial epithelial damage [1]. Sulfasalazine, cholestyramine, budesonide, bismuth preparation or other antidiarrheal and mesalazine have been extensively used in collagenous colitis, and if not successfully treated by those medicines, prednisolone therapy is introduced in most of such cases. Collagenous colitis now has evolved as a fairly common cause of chronic diarrhea in the Western world [1]; however, few cases have been reported in Japan.

In conclusion, we have reported a Japanese patient diagnosed as having collagenous colitis and successfully treated with mesalazine. Further studies are needed to define the efficacy of mesalazine for Japanese patients.

\section{References}

1 Fernández-Bañares F, Salas A, Esteve M, Espinós J, Forné M, Viver JM: Collagenous and lymphocytic colitis. Evaluation of clinical and histological features, response to treatment, and long-term follow-up. Am J Gastroenterol 2003;98:340-347.

2 Nyhlin N, Bohr J, Eriksson S, Tysk C: Systematic review: microscopic colitis. Aliment Pharmacol Ther 2006;23:1525-1534. 